These papers were first presented at the NATO Advanced Research Workshop 'Theoretical Models for Cell to Cell Signalling' held in Knokke-Zoute, Belgium, during September 1988. The Workshop was further supported by the Commission of the European Communities.

\section{Cell to Cell Signalling: From Experiments to Theoretical Models}

\author{
Edited by
}

\section{A. GOLDBETER}

Faculté des Sciences, Université Libre de Bruxelles, Brussels, Belgium

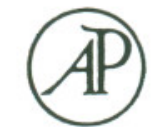

ACADEMIC PRESS

Harcourt Brace Jovanovich, Publishers

London San Diego New York Berkeley Boston

Sydney Tokyo Toronto 


\title{
Pattern formation mechanisms - a comparison of reaction-diffusion and mechanochemical models
}

\author{
J. D. MURRAY ${ }^{a}$ AND P. K. MAINI ${ }^{b}$
}

Centre for Mathematical Biology, Mathematical Institute, 24-29 St Giles', Oxford OX1 3LB, UK

${ }^{a}$ Current address: Applied Mathematics FS-20, University of Washington, Seattle, WA 98195, USA.

${ }^{b}$ Now at: Department of Mathematics, University of Utah, Salt Lake City, UT 84112, USA.

\section{INTRODUCTION}

The elucidation of the underlying mechanisms that occur during morphogenesis is of fundamental importance in developmental biology. From the homogeneous mass of dividing cells of the early blastula, emerge the rich and varied range of pattern and structure observed in animals. Little is known about the mechanisms involved in such development. Although genes must play a key role, genetics says little about the actual mechanisms which produce structure as an organism develops from the egg to its adult form.

Morphogenesis consists of a complex series of chemical, mechanical and electrical interactions and an identification and understanding of these has been the focus of much experimental and theoretical research. Here we briefly compare the two main mathematical models for morphogenetic pattern formation, namely reaction-diffusion systems and mechanochemical models, both of which produce patterns which closely resemble those observed during several developmental processes.

First we outline the theory behind each mechanism and then we focus on the application of these models to only two problems in development, namely, the patterning in the limb that presages cartilage formation and the patterning of feathers and scales. These models have been applied to a 
remarkable array of problems and the reader is referred to the papers listed for other examples. These applications rely on the models' ability to generate spatially heterogeneous solutions. We conclude with a brief discussion of some of the successes, failures and drawbacks of the models.

\section{THE MODEL MECHANISMS}

In his seminal paper on the chemical basis of morphogenesis, Turing (1952) showed that a system of reacting and diffusing chemicals (morphogens) could evolve from an initial homogeneous steady state to a spatially heterogeneous steady state of morphogen concentration. This behaviour is called diffusiondriven instability. If we assume cell differentiation occurs above a certain threshold in chemical concentration, then the landscape of chemical pattern will be reflected in a spatial pattern of cell differentiation.

Numerous reaction-diffusion schemes have since been proposed (for example, Gierer and Meinhardt, 1972; Schnakenberg, 1979) with different reaction kinetics. The general form of the equations for a two-species reaction diffusion system is

$$
\begin{aligned}
& \partial u / \partial t=D_{1} \nabla^{2} u+f(u, v) \\
& \partial v / \partial t=D_{2} \nabla^{2} v+g(u, v)
\end{aligned}
$$

where $u(\mathbf{x}, t)$ and $v(\mathbf{x}, t)$ denote morphogen concentrations at time $t$ and spatial coordinate $\mathbf{x}, D_{1}$ and $D_{2}$ are positive diffusion coefficients, $\nabla^{2}$ is the Laplacian operator and $f(u, v)$ and $g(u, v)$ describe the non-linear reaction kinetics.

The fundamental idea underlying the formation of such chemical prepatterns can be couched in terms of short-range activation and long-range inhibition and is most easily seen in the Gierer-Meinhardt (1972) model kinetics. One of the morphogens, say $u$, may be thought of as an activator. It is produced locally, for example by autocatalysis, and diffuses slower than the other chemical, $v$, which plays the role of inhibitor. If the parameters of the system lie in the appropriate domain - the Turing domain (see Murray, 1982) - this interaction can lead to spatially patterned peaks of activator chemical concentration. This chemical prepattern provides the bauplan for cell differentiation and is the basis for Wolpert's positional information theory (see, for example, Wolpert, 1981) in which cells interpret the chemical prepattern and differentiate accordingly.

Reaction-diffusion mechanisms have been widely studied both mathematically and experimentally. The mathematical analysis has concentrated on the pattern-producing abilities of such systems (see, for example, the books by Britton, 1986; Murray, 1989, the former of which is more mathematical with the latter blatantly applications oriented). Equation systems like (1) and
(2) can produce a large range of spatially heterogeneous patterns, spiral patterns and travelling wave solutions. Much experimental work has been involved in trying to identify the morphogens so as to provide some experimental justification for a reaction-diffusion basis of morphogenesis. So far this has met with limited success.

In the 1980s a fundamentally different approach to cell patterning was proposed by Oster, Murray and their colleagues (for example, Oster et al., 1983; Murray and Oster, 1984a,b; Oster et al., 1985). They modelled the mechanochemical interaction of a major class of cells, known as fibroblasts, with the substratum, the extracellular matrix (ECM), on which they move. The Oster-Murray approach consists of three equations which describe, respectively, cell and matrix conservation and the mechanical equilibrium between cell and matrix. Each equation describes the physico-chemical processes occurring in the cell-matrix milieu. An important difference between the mechanochemical (MC) and reaction-diffusion (RD) models is that the constituent variables and properties involved in the $\mathrm{MC}$ mechanisms are all well known and the parameters in principle measurable. The assumptions behind each equation are the following.

\section{Cell equation}

The general form of the cell equation is

$$
\partial n / \partial t=-\nabla \boldsymbol{J}+F(n)
$$

where $n(\boldsymbol{x}, t)$ is the cell density at time $t$ and position $\boldsymbol{x}$ and $\boldsymbol{J}$ is the cell flux. The second term on the right hand side models cell mitosis, a reasonable form of which is logistic growth

$$
F(n)=r n(N-n)
$$

where $N$ is a typical homogeneous cell density and $r N$ the linear mitotic rate of cells.

Several factors contribute to the cell flux term, for example,

(a) Convection. Cells may ride passively on the ECM, pulled by the forces exerted by their neighbours. This contributes a term of the form

$$
\boldsymbol{J}_{\mathrm{c}}=n \partial \boldsymbol{u} / \partial t
$$

where $\boldsymbol{u}(\boldsymbol{x}, t)$ is the displacement of a material point initially at $\boldsymbol{x}$.

(b) Haptotaxis. Cells move by attaching their filopodia to adhesive sites on the external substratum. It is well known from in vitro studies that cells tend to move up a gradient in adhesive site density (see references in 
Oster et al., 1983). This is known as haptotaxis and may be modelled, in its simplest form, by

$$
J_{\mathrm{h}}=\alpha n \nabla \rho
$$

where $\rho(x, t)$ is the ECM density and $\alpha$ is the (positive) constant coefficient of haptotaxis.

(c) Chemotaxis. Movement of cells up a gradient in some chemoattractant. This type of behaviour is common in many types of cell (for example, in the slime mould Dictyostelium discoideum) and may be modelled, in its simplest form, by

$$
J_{\mathrm{ch}}=\alpha^{\prime} n \nabla \chi
$$

where $\chi(x, t)$ is the concentration of chemoattractant and $\alpha^{\prime}$ is the positive constant coefficient of chemotaxis. Note that inclusion of chemotaxis would necessitate having an equation to describe the behaviour of the chemoattractant $\chi(x, t)$.

(d) Diffusion. Cells may move randomly. This contributes a term

$$
J_{\mathrm{D}}=-D \nabla n
$$

where $D$ is the positive diffusion coefficient.

Various other physical processes which might be involved in development can also be incorporated: a full pedagogical discussion is given in the book by Murray (1989).

\section{Matrix (ECM) equation}

The matrix moves by convection caused by forces exerted by cells and its density is described by the equation

$$
\partial \rho / \partial t=-\nabla \cdot(\rho \partial \boldsymbol{u} / \partial t)
$$

In situations where the cells are secreting matrix, this equation has to be augmented by a matrix source term on the right hand side.

\section{Mechanical force balance equation}

The cell-matrix milieu is modelled as a linear, viscoelastic material with low Reynolds number which implies inertial terms are negligible in comparison with viscous terms and the material is in mechanical equilibrium. The equation of motion is thus of the form

$$
\nabla \cdot \boldsymbol{\sigma}+\rho \boldsymbol{F}=0
$$

where $\boldsymbol{\sigma}$ is a stress tensor and $\rho \boldsymbol{F}$ is an external body force which comes from the surrounding tissue. As well as the viscoelastic contribution from the matrix to $\sigma$, there are several other factors to be taken into account, for example:

(a) Cell traction. In vitro, it is known that mesenchymal (fibroblast) cells exert large traction forces which deform the matrix (Harris et al., 1980). At high cell densities the traction force per cell decreases due to contact inhibition.

(b) Osmotic pressure. In certain cases, for example, in the developing limb bud, the ECM is composed of materials, such as glycosaminoglycans which can exert high osmotic pressure.

In some cases the cell-matrix composite is tethered by elastic-type fibres to some external layer and this must be accounted for in the body force $\rho F$.

Investigations of the pattern-forming capabilities of such MC models (see, for example, Murray and Oster, 1984a,b; Perelson et al., 1986; Maini and Murray, 1988) show that they can produce stable spatially heterogeneous patterns and exhibit travelling wave solutions similar to those obtained from the RD approach. The study of MC models are only at a preliminary stage and, in view of their tensor structure, it is almost certain that they can produce richer pattern structures and a wider range of phenomena than RD systems. From MC theory, the patterning process can also be couched in terms of local short-range activation, initiated by the cell traction, and longrange inhibition but where these are now in terms of forces. A simple detailed intuitive and mathematical description of lateral inhibition models in development is given in Murray (1989).

From a mathematical point of view the onset of a spatially heterogeneous pattern is broadly similar in both models. As a certain parameter increases beyond a critical value, the uniform steady state loses stability and evolves to a spatially heterogeneous pattern. Several parameters can play the role of this bifurcating parameter, for example the relative rates of various chemical reactions or the ratio of diffusivities in RD systems and the cell traction parameter in the MC models. Scale and geometry of the domain also play an important role. The larger the scale, the richer are the possible patterns.

Let us now consider two specific practical problems each mechanism has addressed.

\section{CHONDROGENESIS}

The vertebrate limb has played a major role in embryological and evolutionary studies not only because of its morphological diversification but also because it is one of the easier systems to study experimentally. The 
mechanism that initiates condensations is still unknown. Figure 1 illustrates the process of development of the early limb bud. Cells are produced in the progress zone, the area at the tip of the limb bud, and as they move out of this area they condense and these cell aggregations eventually form cartilage. As development proceeds the skeletal pattern becomes more complex as the limb bud grows, widens and flattens.

The RD approach to explain this spatial patterning phenomenon is essentially the following. As cells leave the progress zone they secrete morphogens which initially form a uniform distribution. As one of the model parameters passes through a bifurcation value the uniform state becomes unstable and evolves to a morphogen concentration pattern with a single central peak. Cells in the centre of the limb bud interpret this peak as the cue to become pre-cartilage cells. As the scale and geometry of the limb bud change, the RD mechanism can produce more complex patterns which can account for the later condensation patterns.

There are several MC scenarios. That in Oster et al. (1985) has the cells move out of the progress zone, secrete hyaluronic acid (HA), a highly osmotic glycosaminoglycan, which swells the limb bud and initially keeps the cells apart. Prior to condensation, it is known that cells secrete hyaluronidase, the enzyme which degrades HA. The model suggests that this could

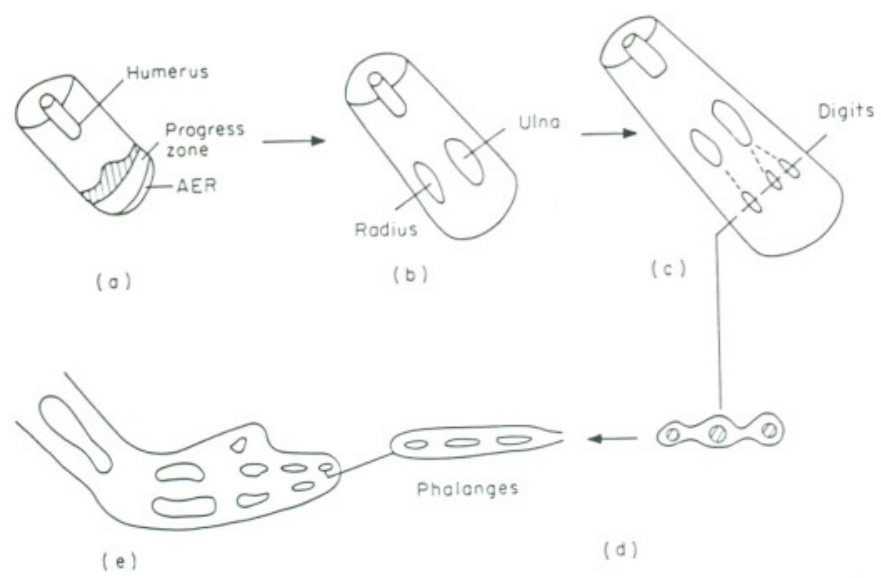

1. A scenario showing a possible pattern of bifurcations according to the . A MC model lo apical ectodermal ridge AER) (AER), aggregate and condius and ulna. (c) The asymmetry induced in the limb geometry by cell tractions leads to a 列 (d) The limb bud is now flattened and the digits bifurcate longitudinally to give the phalanges. (e) Sketch of the full chick limb skeletal pattern. lead to partial osmotic collapse of the internal limb bud which brings cells in closer proximity to their neighbours. The short-range traction forces then take over and can induce a bifurcation wherein the uniform distribution of cells evolve to one where cells aggregate in the centre. These cells then condense to form cartilage. As with the RD system, scale and geometry effects can account for subsequent pattern.

Although the mathematical theory behind pattern formation is similar for both mechanisms, the biological scenarios are quite different. RD relies on a number of discrete steps which effectively form an 'open loop' system (which is notoriously unstable):

(i) The formation of a prepattern of morphogen concentration.

(ii) The interpretation of this morphogen concentration by cells.

(iii) A change in limb scale and geometry leading to the more complex structures.

The MC approach relies on fewer steps. The cellular pattern is formed directly by the process of aggregation - not a prepattern. This cell aggregate has the ability to deform the geometry of the limb bud thus inducing subsequent bifurcations (Oster et al., 1983). Since mechanical deformation can alter the transcription of cells the MC model is not an open-loop system; it has the ability for self-correction. The RD approach does not address the question of the change in geometry and scale of the developing limb bud.

Several experiments have been performed to try and test the above mechanisms. From the RD viewpoint the first essential step is to identify the morphogens. To date this has met with only limited success. Calcium and retinoic acid (RA) have been proposed as two possible candidates but the evidence is controversial. Indeed, in the case of RA, experiments by Kochar et al. (1984) suggest that its effects may be due to the observation that RA greatly changes the ability of cells to synthesize HA. This tends to substantiate an MC approach.

The main body of experimental work centres around grafting experiments. For example, if cells from the region known as the zone of polarizing activity (ZPA), which is located in the posterior part of the limb bud, are grafted onto the anterior of the limb, the skeletal elements are duplicated. It is known that such grafting experiments result in abnormal widening of the limb bud (Smith and Wolpert, 1981). Both the above mechanisms would thus predict an increase in richness of pattern due to the increase in scale.

Alberch and Gale (1983) treated developing frog limb buds with colchicine, a mitotic inhibitor. They found that the resulting limb usually had one or more digits missing and was smaller in size than a normal limb. This, again is consistent with both the above mechanisms - a decrease in size resulting in a decrease in pattern. Further results and a comprehensive theory of limb development has been given by Oster et al. (1988).

A major criticism of RD theory is that it is very sensitive to initial and 
boundary conditions and to parameter values. This is obviously a major drawback to any mechanism proposed for a robust developmental process such as chondrogenesis. Arcuri and Murray (1986) partially answered this criticism when they showed that with fixed concentrations on the boundary, RD systems became less sensitive. In the limb bud, the sleeve of HA already provides such a fixed boundary condition in the MC models.

Recent graft experiments by Wolpert (personal communication) seem to indicate that there is indeed a prepattern formed before any variation in cell densities are evident. This would certainly be a major point in favour of a RD theory.

\section{SKIN ORGAN FORMATION}

Vertebrate skin forms many specialized structures, such as hair, scales, feathers and glands. A widely studied problem of considerable interest is the generation of feather germs in chicks (see, for example, Davidson, 1983). These structures are distributed across the surface of the animal in a characteristic and regular fashion. As with other organs the developmental processes involved are not understood. This is currently a problem of considerable interest since its solution would be a milestone in understanding embryogenesis.

The chick skin consists of an epidermal layer of columnar, non-motile cells overlying a dermal layer composed of motile, mesenchymal (fibroblast) cells in a collagenous extracellular matrix. Each feather germ, or primordium, consists of a thickening of the epidermis, called a placode, and a condensation of dermal cells, called a papilla. Davidson (1983) found that in the chick, initially a primordium became visible in the centre of the dorsal midline. A row of primordia then appeared along the dorsal midline. Subsequent rows of primordia then formed on either side of this initial row at intermediate points: the pattern spread laterally forming a hexagonal array.

Perelson et al. (1986) solved a MC model in one dimension and showed that, as cell traction increased, an initially uniform distribution of cells could evolve to a pattern of aggregations. This pattern of cells produces a strain field which causes cells in neighbouring rows to aggregate at interdigitating points, thus leading to hexagonal structure as shown in Fig. 2.

It is not easy to reconcile RD theory with such sequential pattern formation. The mechanism would have to rely on forming a prepattern, to which cells become competent to respond in a sequential manner. The process by which pattern forms on the dorsal midline suggests some prepattern mechanism which determines the initial central condensation. However, Davidson (1983) took a section of chick pteryla on which pattern was forming and cut ahead of the 'wave' of laterally expanding primordia. $\mathrm{He}$ found that primordia formed in the skin in front of the cut but their

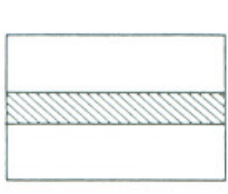

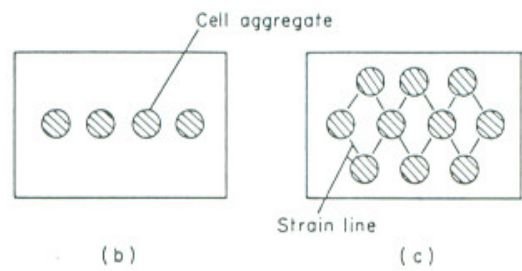

Fig. 2. Scenario for hexagonal pattern formation in the chick back according to MC theory. (a) Section of the chick back illustrating uniform cell density along the dorsal midline. As these cells mature, their traction increases and the uniform steady state loses stability and evolves to a heterogeneous spatial pattern of cell aggregations (b). This line of aggregates imposes a strain field causing aggregation along neighbouring rows at intermediate points leading to hexagonal patterning $(\mathrm{c})$

position was random compared to the previous row behind the cut. This argues against a prepattern theory

Both RD and MC theories make predictions on the spacing between primordia. The RD prediction is in terms of diffusion coefficients and reaction rates for chemicals which have yet to be identified and therefore such predictions cannot be tested. The MC approach predicts that decreasing the total cell number will increase the spacing (Oster et al., 1983). Davidson (personal communication) irradiated a section of dorsal pteryla, decreasing the total cell number, and found that the spacing did indeed increase.

Both the above mechanisms simply concentrate on the dermal pattern. Dermal-epidermal recombination experiments strongly suggest that there is interaction between both layers. Nagorcka et al. (1987) proposed a hybrid model as a mechanism for scale pattern formation in reptiles. Their model consisted of a two-component RD system of epidermal origin coupled to a cell traction (MC) model of dermal origin. They showed that such a mechanism could produce a pattern consisting of two different sized scales, with correspondingly different interscalar spacings, superimposed on each other. Their patterns closely resemble those observed in certain reptiles such as the armadillo. Coupled MC systems could also produce such patterns.

\section{DISCUSSION}

In the last two sections we focused on the ability of RD and MC systems to generate spatially heterogeneous solutions and showed how they could be considered as possible mechanisms for certain developmental processes. They have also been proposed as possible mechanisms for several other developmental phenomena, for example, butterfly and moth wing patterns (Murray, 1981) in the case of RD theory and, in the case of MC theory, 
movements of epidermal cell sheets during the process of gastrulation (Odell et al., 1981), contraction waves on the surface of vertebrate eggs just after fertilization (Cheer et al., 1987; Lane et al., 1987) and others (see, for example, Murray, 1989). In a broader sense, RD theory has been proposed as the mechanism which accounts for certain regeneration processes, for example in the alga Acetabularia (Goodwin et al., 1985) where calcium is proposed as the morphogen: quantitative experimental evidence is presented to support this hypothesis. RD has definitively been shown to be the mechanism in the case of spiral wave phenomena in slime mould Dictyostelium (see, for example, the brief review by Tyson and Murray, 1989). MC theory has been applied to the process of wound healing (Murray et al., 1988): qualitative comparison of the theory with experiment is encouraging. The list of proposed applications is now very large.

An important feature of all of these models is the effect of geometry and scale. Oster et al. (1988) used such ideas to help make more precise the notion of developmental constraints in the vertebrate limb. They showed that, with only a limited number of allowable condensation patterns, one could use the effects of scale and geometry to 'construct' the entire limb. Their results have important consequences for evolution, which suggest that mutation can only give rise to a limited variety of different limb skeletal structure from a normal limb.

Although both theories have very rich pattern formation structure, clearly both suffer from major drawbacks. The existence of morphogens is, in most cases, still a controversial issue. The problem of how cells interpret a prepattern cannot begin to be addressed until they are identified. RD mechanisms are of 'open-loop' type, that is, once the prepattern is laid down, the eventual morphogenetic structure is fixed - there can be no feedback from the environment to modify the pattern. Such open-loop systems are unstable and seem an unreliable mechanism for many developmental processes which are, in the main, robust and self-correcting.

$\mathrm{MC}$ theory is based, to a large extent, on known cellular properties from in vitro studies. It is a controversial issue as to whether certain of these properties are exhibited by cells in vivo. In some cases of skin organ formation it is unclear if cells do actually form aggregations.

The ability of a model to mimic patterns observed in a developmental process is only the first, but necessary step to its candidacy as a possible mechanism. A model must be able to make testable predictions. From this point of view many predictions of RD theory are hard to verify because they are in terms of properties of morphogens yet to be identified, whereas those of MC theory are based on parameters which are measurable.

The processes that occur in development are clearly highly complex. Mathematical models are, necessarily, based on gross simplification. However, they do tend to focus on particular elements of the process and help determine the role played by such elements in the overall process. The role of theory is to provide a conceptual framework for the experimenter and to make predictions which encourage, or provoke, experimentation. From this point of view, mathematical models for development are fulfilling their role and will certainly play a significant part in the understanding of one of the major problems in science.

Acknowledgements: P.K.M. would like to thank the Science and Engineering Research Council of Great Britain for its financial support through a grant to the Centre for Mathematical Biology, University of Oxford.

\section{REFERENCES}

Alberch, P. and Gale, E. (1983) Size dependency during the development of the amphibian foot. Colchine induced digital loss and reduction. J. Embryol. exp. Morph. 76, 177-97.

Arcuri, P. and Murray, J. D. (1986) Pattern sensitivity to boundary conditions in reaction-diffusion models. J. Math. Biol. 24, 141-65

Britton, N. F. (1986) Reaction-Diffusion Equations and their Applications to Biology. Academic Press, New York.

Cheer, A., Nuccitelli, R., Oster, G. F. and Vincent, J.-P. (1987) Cortical activity in vertebrate eggs I: The activation waves. J. theor. Biol. 124, 377-404

Davidson, D. (1983) The mechanism of feather pattern development in the chick. I The time determination of feather position. II. Control of the sequence of pattern formation. J. Embryol. exp. Morph. 74, 245-73.

Gierer, A. and Meinhardt, H. (1972) A theory of biological pattern formation. Kybernetik 12, 30-9.

Goodwin, B. C., Murray, J. D. and Baldwin, D. (1985) Calcium: the elusive morphogen. In Acetabularia. Proc. 6th Intern. Symp. on Acetabularia (eds S.

Harris, A. K., Ward, P. and Stopak D. (1980) Silicone rubber substrata: A new wrinkle in the study of cell locomotion. Science, N.Y. 208, 177-9.

Kochar, D. M., Penner, J. D. and Hickey, T. (1984) Retinoic acid enhances the displacement of newly synthesized hyaluronate from cell layer to culture medium during early phases of chondrogenesis. Cell Diff. 14, 213-21.

Lane, D. C., Murray, J. D. and Manoranjan, V. S. (1987) Analysis of wave phenomena in a morphogenetic mechanochemical model and an application to post-fertilisation waves on eggs. IMA J. Math. appl. Med. Biol. 4, 309-31.

Maini, P. K. and Murray, J. D. (1988) A nonlinear analysis of a mechanical model for biological pattern formation. SIAM J. appl. Math. 48, 1064-72.

Meinhardt, H. (1982) Models of Biological Pattern Formation. Academic Press, London.

Murray, J. D. (1981) On pattern formation mechanisms for Lepidopteran wing patterns and mammalian coat markings. Phil. Trans. R. Soc. Lond. B 295, 473-96.

Murray, J. D. (1982) Parameter space for Turing instability in reaction diffusion mechanisms: a comparison of models. J. theor. Biol. 98, 143-63.

Murray, J. D. (1989) Mathematical Biology. Springer-Verlag, Berlin

Murray, J. D. and Oster, G. F. (1984a) Generation of biological pattern and form. IMA J. Math. appl. Med. Biol. 1, 51-75. 
Murray, J. D. and Oster, G. F. (1984b) Cell traction models for generating pattern and form in morphogenesis. J. Math. Biol. 19, 265-79.

Murray, J. D., Maini, P. K. and Tranquillo, R. T. (1988) Mechanochemical models for generating biological pattern and form. Phys. Rep. 171, 59-84.

Nagorcka, B. N., Manoranjan, V. S. and Murray, J. D. (1987) Complex spatial patterns from tissue interactions - an illustrative model. J. theor. Biol. 128, 359-74.

Odell, G. M., Oster, G. F., Burnside, B. and Alberch, P. (1981) The mechanical basis of morphogenesis I: Epithelial folding and invagination. Devl Biol. 85, 446-62.

Oster, G. F., Murray, J. D. and Harris, A. K. (1983) Mechanical aspects of mesenchymal morphogenesis. J. Embryol. exp. Morph. 78, 83-125.

Oster, G. F., Murray, J. D. and Maini, P. K. (1985) A model for chondrogenic condensations in the developing limb: the role of extracellular matrix and cell tractions. J. Embryol. exp. Morph. 89, 93-112.

Oster, G. F., Shubin, N., Murray, J. D. and Alberch, P. (1988) Evolution and morphogenetic rules. The shape of the vertebrate limb in ontogeny and phylogeny. Evolution 42, 862-84.

Perelson, A. S., Maini, P. K., Murray, J. D., Hyman, J. M. and Oster, G. F. (1986) Nonlinear pattern selection in a mechanical model for morphogeneșis. J. Math. Biol. 24, 525-41.

Schnackenberg, J. (1979) Simple chemical reaction systems with limit cycle behaviour. J. theor. Biol. 81, 389-400.

Smith, W. R. and Wolpert, L. (1981) Pattern formation along the anteroposterior axis of the chick wing: the increase in width following a polarizing graft and the effect of X-irradiation. J. Embryol. exp. Morph. 63, 127-44.

Turing, A. M. (1952) The chemical basis of morphogenesis. Phil. Trans. R. Soc. Lond. B 237, 37-72.

Tyson, J. J. and Murray, J. D. (1989) Cyclic-AMP waves during aggregation of Dictyostelium amoebae. Development (in press).

Wolpert, L. (1981). Positional information and pattern formation. Phil. Trans. R. Soc. Lond. B 295, 441-50. 